\title{
The structure of turbulent flow behind the NACA 0012 airfoil at high angles of attack and low Reynolds number
}

\author{
Vitalii Yanovych ${ }^{1,2 *}$, Daniel Duda ${ }^{1}$, Václav Uruba ${ }^{1,2}$, and Pavlo Kosiak ${ }^{1}$ \\ ${ }^{1}$ Department of Power System Engineering, Faculty of Mechanical Engineering, University of West \\ Bohemia in Pilsen, Univerzitní 22, 30614 Pilsen, Czech Republic. \\ ${ }^{2}$ Department of Fluid Dynamics, Institute of Thermomechanics, Czech Academy of Sciences, \\ Dolejškova 5, 182 00, Prague, Czech Republic.
}

\begin{abstract}
This paper shows the results of a study of the turbulent structure behind the NACA 0012 airfoil. During the experiment, the flow velocity was $20 \mathrm{~m} \cdot \mathrm{s}^{-1}$. That corresponds the Reynolds number $1.3 \cdot 10^{5}$. The point behind the trailing edge was chosen for experimental studies. Measurements were performed at six angles of attack $\alpha=0^{\circ}, 15^{\circ}, 30^{\circ}, 45^{\circ}, 60^{\circ}, 75^{\circ}$ and various cases of positioning the measuring section. Namely at a constant crosssection and a constant distance behind the airfoil. The NetScanner pressure system and hot-wire technique were used for experimental studies. The obtained data allowed us to investigate the wake topology. The average velocity and standard deviation distributions are clearly grouped depending on the angle of attack. Thus, flowing around the airfoil is better up to $\alpha \leq 15^{\circ}$. Distributions by power density and dissipation spectra also have a similar grouping tendency. Finally, we investigated the turbulent structure according to the research program. We found that at $\alpha \geq 45^{\circ}$, depending on the measurement case, there is a clear difference in the distribution of standard deviation, Eulerian length scale, Taylor micro-scale, and Reynolds number based on the Taylor micro-scale. The obtained values at the constant distance, in contrast to the constant cross-section, are reduced.
\end{abstract}

\section{Introduction}

The study of the flow structure around an airfoil is a common task. Because even the smallest changes in their geometry play a key role in the overall evolution of the streamlined flow. In recent years, NACA family profiles have become widespread. They have a great variety and are actively used for wind energy, aviation, and energy systems.

It should be noted that the NACA 0012 profile deserves special attention among many profiles. This is because it has a relatively simple shape geometry and high aerodynamic characteristics. Which makes it a benchmark not only for practical uses but also for a variety of experimental and analytical studies. That is why many papers are devoted to the study of the flow structure around this aerodynamic profile. For example, the distribution of pressure

\footnotetext{
* Corresponding author: yanovych@kke.zcu.cz
} 
profile and wake profile behind airfoil is very well described in [1]. Based on the obtained experimental data, the authors found the coefficients of lift, drag, and torque twist. In addition, they found that the use of a gurney flap can increase the lift rate by $25 \%$. Similar studies are also shown in the papers [2-4]. Where the authors focused on the study of aerodynamic coefficients and features of the wake topology behind airfoil. The investigations were performed at angles of attack up to $40^{\circ}$. While the paper [5] shown the results of the study of the flow evolution around the airfoil up to $90^{\circ}$. But with a relatively high degree of turbulence intensity of the overall flow.

Frequently NACA 0012 airfoil is used as a basis for analyzing the shape effect of the trailing edge on the structure of the turbulent flow. For example, [6] shows a study of the effect of vortex instability on the aerodynamic characteristics of the airfoil with multiscale modifications of its trailing edge. The authors concluded that large multi-scale trailing edges at some distance from each other break down the vortex shedding mechanism. That improves the aerodynamic characteristics of the airfoil and reduces the amount of noise generated. It should be noted that the latter is a key issue in the development of modern wind turbines. This phenomenon is well described in the paper [7]. Where the authors conducted a comparative analysis of experimental studies of noise generated by stationary and rotating blades of the wind turbine rotor.

Nowadays, computational fluid dynamics (CFD) is used in many cases to better understanding flow dynamics. The NACA 0012 airfoil is usually used to compare the aerodynamics of the streamlined body or to test the flow simulation algorithm around them. For example, the authors [8] based on numerical simulations showed a comparative assessment of aerodynamic characteristics between S809 and NACA 0012. Whereas the paper [9] presents the results of the numerical investigation of the separation mechanisms and prediction of the turbulence transition in a separate shear layer behind symmetric airfoil.

Generally, much of the papers about NACA 0012 airfoil show the study of aerodynamic coefficients and flow topology at angles of attack that do not exceed critical values. However, studying the structure of the flow around the airfoil at a high angle of attack is an important task also. That is especially true for aviation. For example, at high and low altitudes, where even small maneuvers may require high angles of attack. In the first case due to the low density of air in the upper atmosphere. In the second at relatively low flight speed. In addition, when landing, all aircraft use flaps at a high angle of attack. Thus, for military aircraft, a high angle of attack can significantly increase their maneuverability. Accordingly, the F-18 HARV, Su-27M, or Su-35 aircraft can use angles of $70^{\circ}, 120^{\circ}$, and $180^{\circ}$, respectively, for their maneuvers.

Many researchers are using hot-wire to study the structure of turbulence around an airfoil. Basically, they perform measurements at some cross-sections behind a bluff body. Even at different angles of attack, the distance remains the same. However, as the angle of attack increases, the distance between the trailing edge and the measuring section growing also. As a result, the structure of the turbulent flow, on the way to the measuring point evolve more. The main idea of this paper is to investigate this evolve behind the symmetric profile of NACA 0012.

\section{Experimental facilities}

The experiment was conducted in a low-speed open-type wind tunnel at the Department of Energy Systems Engineering, West Bohemian University [10, 11]. The internal dimensions of the width, height, length of the test section were $0.3 \mathrm{~m}, 0.2 \mathrm{~m}$ and $0.75 \mathrm{~m}$, respectively.

To study the structure of turbulent flow behind an asymmetrical airfoil, the profile NACA 0012 was chosen. It is a typical outboard airfoil for utility-scale wind turbines. The original dimensions of the airfoil were the chord length $c=100 \mathrm{~mm}$, the span $L \cdot c^{-1} \approx 1$ and 
the thickness $b \cdot c^{-1} \approx 0.12$. At these parameters, the blockage ratio, relevant to the test section, was a proximally $2 \%$ at an attack angle $\alpha=0^{\circ}$. The airfoil was manufactured of plastic using 3D printing technology. We used a PRUSA i3 printer and PLA thermoplastic filament materials for this purpose. After that by P2500 sandpaper, we reduced the surface roughness of airfoil to $5 \mu \mathrm{m}$.

To change the angle of attack, the airfoil was attached to the shaft of the stepper motor. Which was placed on the cover of the test section. Thus, the aerodynamic center of the airfoil was at $\Delta z \cdot c^{-1} \approx 1.53$ and $\Delta x \cdot c^{-1} \approx 2.23$. Where $\Delta z$ and $\Delta x$ are the distance to the wall and the inlet of the test section, respectively. The scheme of the experimental investigation is shown in Fig. 1. Measurements were performed at six positions of angles from $\alpha=0^{\circ}$ to $\alpha=75^{\circ}$ in steps of $15^{\circ}$. Accordingly, at $\alpha=0^{\circ}$ the airfoil chord has the same direction as the general flow. The Reynolds number was based on the length of the airfoil chord and was equal to $1.3 \cdot 10^{5}$. That corresponds to the flow velocity of $20 \mathrm{~m} \cdot \mathrm{c}^{-1}$.

The 9116 series NetScanner pressure system and the five-hole Pitot tube were used to estimate the wake topology depends on the angle of attack. The measurements took place in the plane behind the airfoil at $l_{1} \cdot c^{-1} \approx 0.28$ at step $w_{z} \cdot c^{-1} \approx 0.05$.

The 55P15 hot-wire probe was used to study the $u$-component of turbulent fluctuation behind airfoil. The position of the measuring point according to the experimental procedure was changing by the traverse system. The hot-wire signal was sampled at $75 \mathrm{kHz}$. While the measurement time at one point was $10 \mathrm{~s}$. We also used filtering for the received signal: for the High Pass - $10 \mathrm{~Hz}$ and the Low Pass - $30 \mathrm{kHz}$.

Of particular interest in these studies was the structure of turbulence directly behind the trailing edge. That is why in the range $r \cdot c^{-1} \approx 0.28$ the distance between the measuring points was smaller $k_{z} \cdot c^{-1} \approx 0.02$ than outside $k_{z} \cdot c^{-1} \approx 0.05$. In the general case, the measuring crosssection from $z \cdot c^{-1} \approx-1.2$ to 0.8 was located at a height $y \cdot c^{-1} \approx 1.8$. The latter value also corresponds to the center of span airfoil. While the location of the cross-section was different. In the first case, the position remains unchanged relative to the coordinates of the test section. Thus, at a growing angle of attack, the distance from the measuring cross-section to the airfoil increased. Whereas in the second case, it was contrary. The distance to the trailing edge always stayed the same $l_{2} \cdot c^{-1} \approx 0.15$.
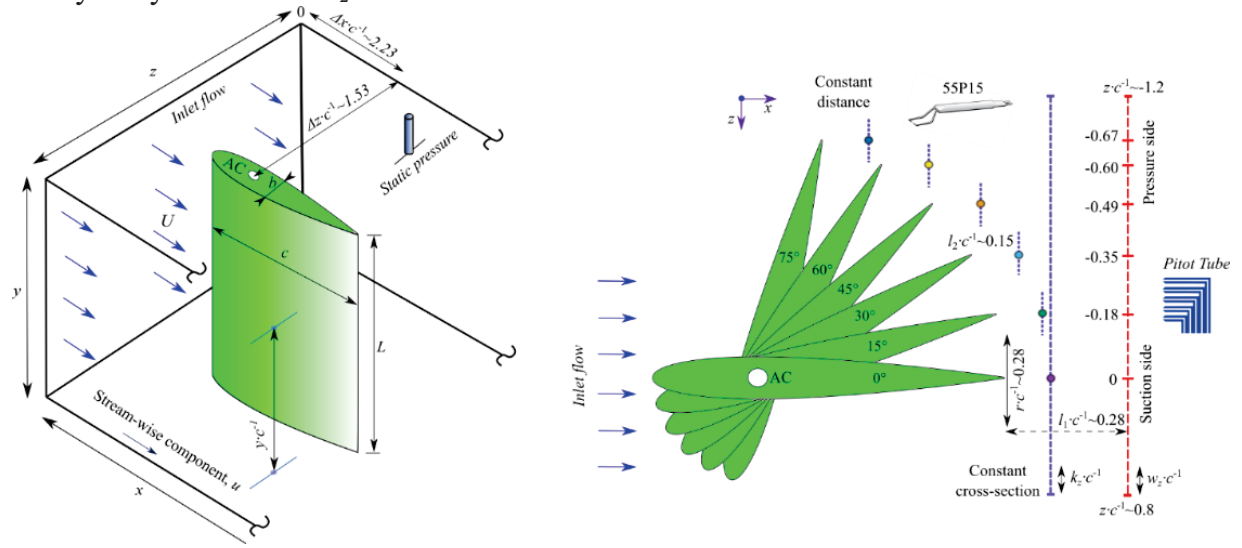

Fig. 1. Scheme of the experimental investigation. The left shows the positioning of the airfoil in the test section. The arrows in blue indicate the direction of airflow $\mathrm{U}$. Where $\mathrm{z} \cdot \mathrm{c}^{-1}$ and $\mathrm{x} \cdot \mathrm{c}^{-1}$ respectively are the width and length of the measuring fields. The $\mathrm{k}_{\mathrm{z}} \cdot \mathrm{c}^{-1}, \mathrm{w}_{\mathrm{z}} \cdot \mathrm{c}^{-1}, \mathrm{y} \cdot \mathrm{c}^{-1}$ and $\mathrm{r} \cdot \mathrm{c}^{-1}$ are the steps between measuring points for hot-wire and NetScanner pressure system, the height of the location and area of the trailing edger, respectively. Whereas $\Delta \mathrm{z} \cdot \mathrm{c}^{-1}$ and $\Delta \mathrm{x} \cdot \mathrm{c}^{-1}$ are the coordinates of the aerodynamic center (AC). Blue and red dashed lines are a set of measuring cross section for hot-wire and pressure system, respectively. 


\section{Results and discussion}

\subsection{Wake topology}

A series of experimental studies were performed using the 9116 series NetScanner system. As a result, we obtained the surfaces of the flow velocity distribution behind the airfoil at a different angle of attack (Fig. 2). This made it possible to evaluate the general characteristic of the wake topology. It was found that with increasing the angle of attack from $0^{\circ}$ to $75^{\circ}$, the width wake increases by 8.5 times. While the wake depth begins to increase sharply after $\alpha=15^{\circ}$ and has the maximum value $U \mathcal{u}^{-1} \approx-0.57$ at $\alpha=45^{\circ}$. The physically negative value of this parameter means the presence of backflow. Also, it should be noted that exactly at $\alpha=15^{\circ}$ we can clearly observe the upper and downer wingtip vortices. Further increase in the angle of attack leads to the disappearance of this feature. This is due to the increase in the area of the velocity deficit behind the airfoil. At the same time, outside this area, the flow velocity also increases. Thus, at $\alpha=75^{\circ}$ the normalized velocity is equal to $U \cdot u^{-1} \approx 1.4$. This pattern is related to the blockage ratio of the airfoil at different $\alpha$.

To further study the structure of the turbulent flow by hot-wire, we chose the point M. Which was placed exactly behind trailing edge. As noted above, the experiments were performed for two cases: at constant cross-section and constant distance.

The experimental results are presented as a series of graphs. They show the distribution of the stream-wise velocity $\mathrm{u}$ and standard deviation $\sigma_{u}$ normalized by average flow velocity $U$ depending on the angle of the attack (Fig. 3 a, b, d, e). For convenience, the resulting curves are located relative to the position of the trailing edge at $\alpha=0^{\circ}$.

The obtained distributions for the two measurement cases at different angles of attack have similar behavior. The obtained curves clearly show the developed of the instability region behind the airfoil. Moreover, relative to the trailing edge, this region is expanding in direction to the suction side. While at the boundaries of the wake the values of $u \cdot U^{-1}$ and $\sigma_{u} \cdot U^{-1}$ is growing. But unlike $u \cdot U^{-1}$ the distribution of $\sigma_{u} \cdot U^{-1}$ has two local peaks. The largest of them is shifted to the pressure side. It is because at positive angle $\alpha$, the vortices which passing from the suction side interact with the vortices from the pressure side. This leads to the emergence of a dominant vortex in the general flow. Whereas at $\alpha=0^{\circ}$, as a result of delaying boundary layer separation, this feature disappears. In general, sharp changes in the wake topology are observed after $\alpha \geq 30^{\circ}$. While at $\alpha=0^{\circ}$ and $\alpha=15^{\circ}$ the width and depth of the wake remain the smallest. 

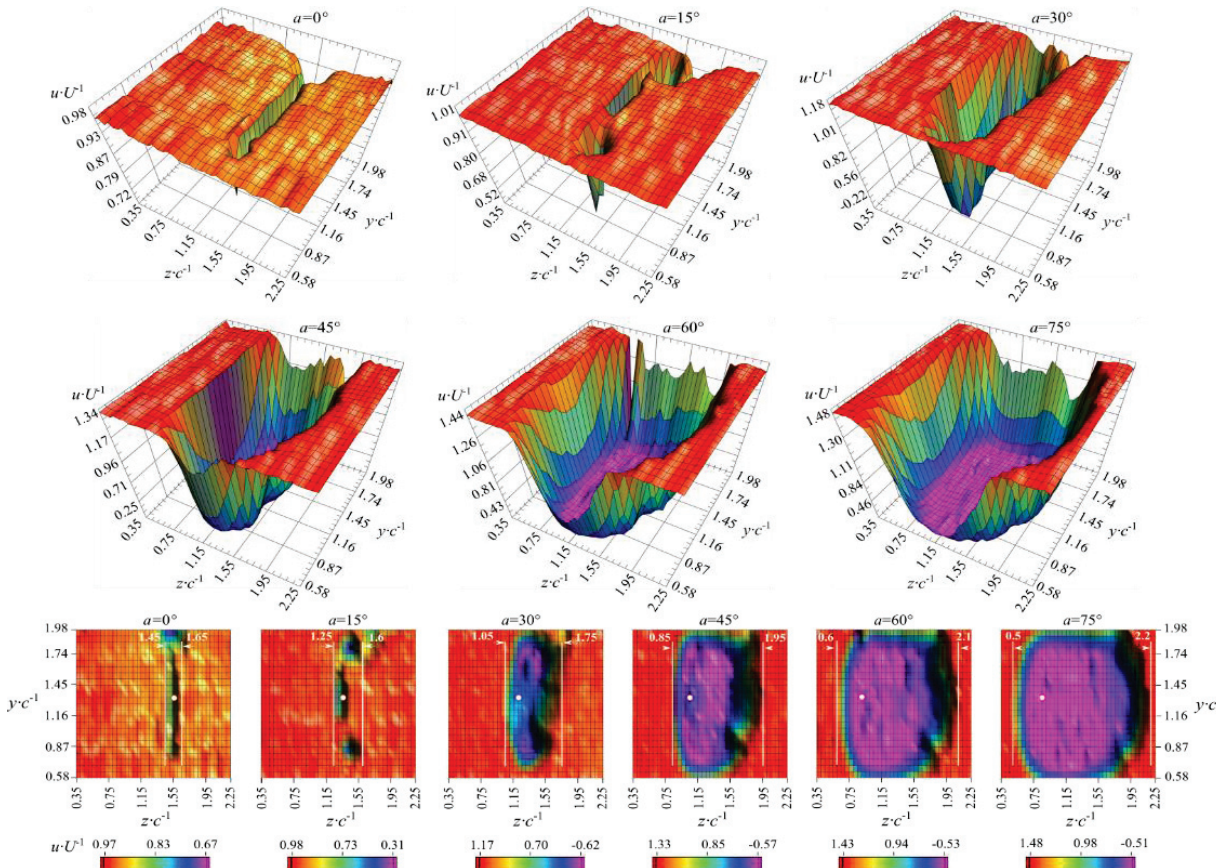

Fig. 2. The distributions of normalized velocity $u \cdot U^{-1}$ depending on the angle $\alpha$ at $1_{1} \cdot \mathrm{c}^{-1} \approx 0.28$ and $\operatorname{Re}$ $\approx 1.3 \cdot 10^{5}$. The upper part shows the flow velocity distribution surface. Whereas below there are its projections on the coordinates $\mathrm{x}$ and $\mathrm{y}$. The white lines outline the velocity deficit area. The point $\mathrm{M}$ is the position of the measuring behind the trailing edge.
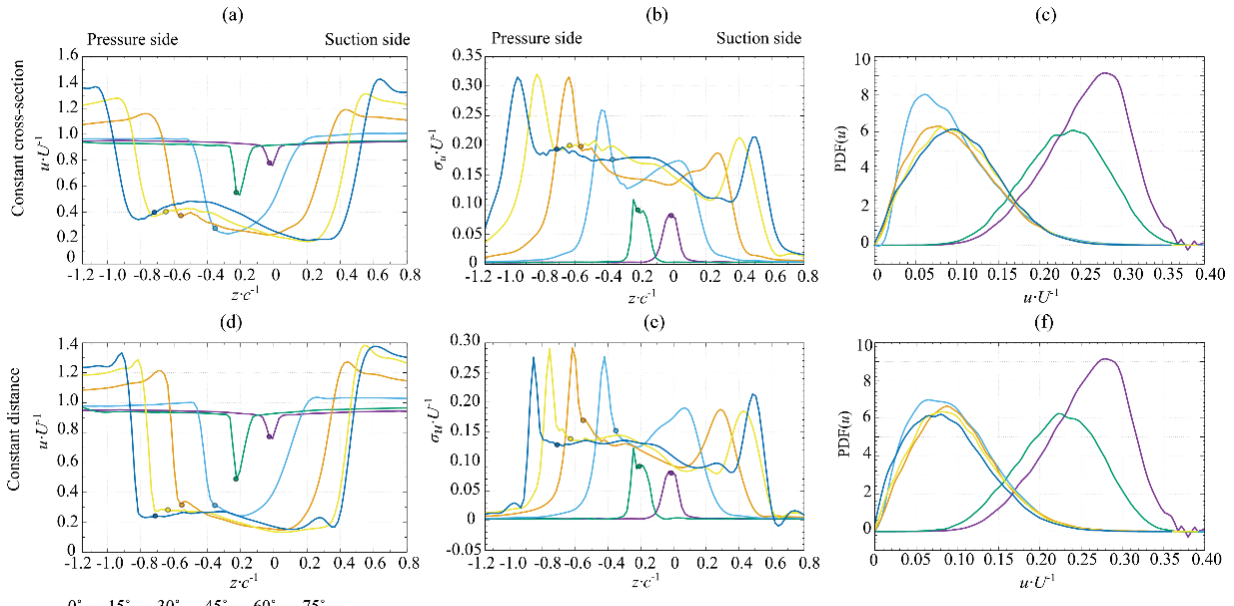

Fig. 3. $(a, b, d, e)$ The normalized velocity $u \cdot U^{-1}$ and standard deviation $\sigma_{u} \cdot U^{-1}$ distribution depending on angle of attack at $1_{1} \cdot \mathrm{c}^{-1} \approx 0.15$ and $\operatorname{Re} \approx 1.3 \cdot 10^{5}$. (c, f) The right two graphs represent probability density functions of the velocity fluctuations $\operatorname{PDF}(\mathrm{u})$ at the measuring point $\mathrm{M}$. The upper $(\mathrm{a}, \mathrm{b}, \mathrm{c})$ and lower (d, e, f) graphs show the flow characteristics measured at constant cross-section and constant distance, respectively. Colored lines and dots respectively reflect the distribution of flow characteristics and the position of the measuring point depending on variation $\alpha$. 
The probability density function $(\mathrm{PDF}(\mathrm{u}))$ was used to describe the stream-wise fluctuation at the measurement points $M$. As generally expected for turbulence, we found that fluctuations are well described by a Gaussian distribution. In addition, it is clearly observed that for both measuring cases the average velocity at $\alpha \leq 15^{\circ}$ is approximately 5 times higher than at $\alpha \geq 30^{\circ}$.

Thus, we can conclude that the flowing around airfoil is better up to $\alpha \leq 15^{\circ}$. Further increase in the angle of attack leads to the development of an instability region there produces active perturbations. This phenomenon has a significant impact on the location of the point where boundary layer separation begins. Many publications are devoted to this issue [12-14]. The researchers note the significant impact of flow separation on the lift and drag characteristics of the airfoil. That is why each airfoil has an optimal angle of attack. For symmetrical airfoil in most cases, it is up to $15^{\circ}$. Where the ratio of lift and drag coefficients will be the highest.

\subsection{The power and dissipation spectra}

Power spectral density was used to determine the distribution of flux energy intensity between different scale components at measuring point $\mathrm{M}$. Graphical interpretation of normalized Eulerian power spectral densities of velocity fluctuations depending on the angle $\alpha$ at different cases of measuring are shown in Fig. $4(a, b)$. For representing the Kolmogorov dimensionless coordinates were used. The obtained spectrum was normalized by $\left(\epsilon v^{5}\right)^{1 / 4}$ and the wave number $k \cdot \eta$. Where $\eta, \epsilon$ and $v$ is dissipation scale, dissipation rate and kinematic viscosity of air $1.5 \cdot 10^{-5}\left[\mathrm{~m}^{2} \mathrm{c}^{-1}\right]$, respectively. The different flow velocity at the measurement point leads to some shift of the obtained curves relative to the wavenumber.

As is known, according to Kolmogorov's law, the spectral density of turbulence energy decreases with increasing wave number. As a result, the resulting curve has a slope $f=-5 / 3$ $[15,16]$. This pattern can be clearly observed for the point $M$ regardless of the angle of attack. It should be noted that the behavior of the obtained curves for the two measuring cases is almost the same. The main difference is observed in the inertial subrange at $\alpha=30^{\circ}$. Physically, it characterizes the range where turbulence kinetic energy is transferred from larger to smaller scales without loss. This is due to the distance between the measuring point and the trailing edge as the angle of attack increases. 
(a)

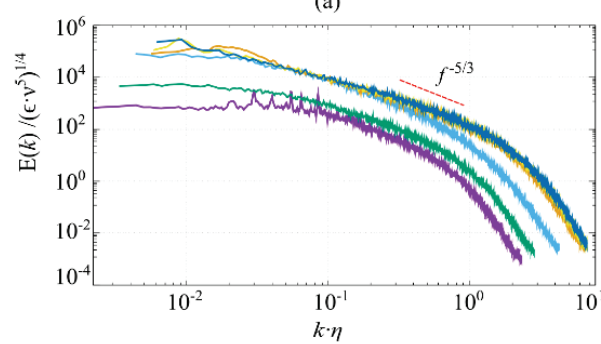

(c)

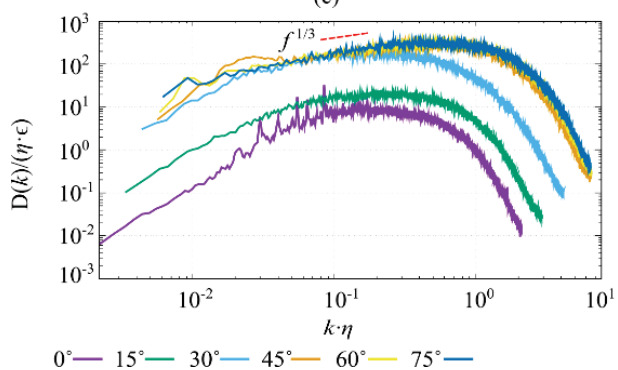

(b)

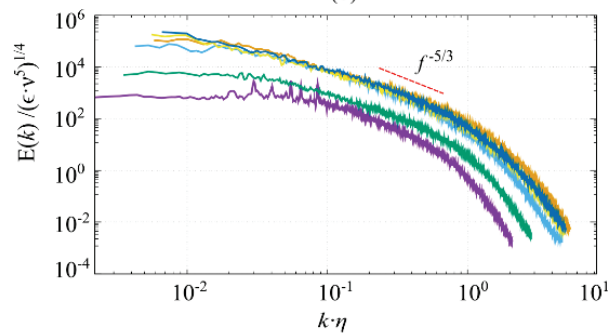

(d)

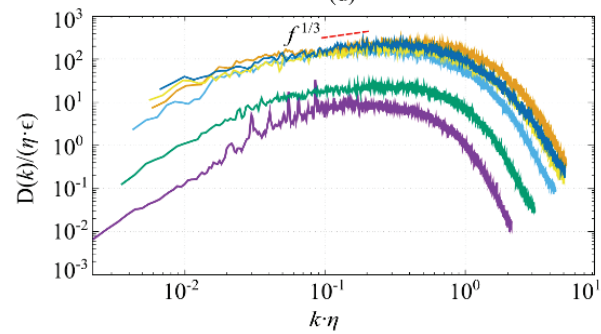

Fig. 4. Normalized Eulerian power $E(k)$ and dissipation $D(k)$ spectral of stream-wise fluctuations at point $\mathrm{M}$ depending on the angle of attack and case of measurement. The right (a, c) and left (b, d) graphs represent the flow characteristics at constant cross-section and at constant distance, respectively. The experimental Reynolds numbers was $1.3 \cdot 10^{5}$. Colored lines reflect the value of the angle $\alpha$.

In both cases of measurement, the received curves can be divided into two groups. Thus, before $\alpha=15^{\circ}$ and after, there is a clear difference in the energy characteristics of the flow fluctuations. Moreover, for high angles of attack at $\alpha \geq 30^{\circ}$, it is almost the same.

In addition, the dissipation spectrum for the measuring points $M$ was found (Fig. $4 \mathrm{c}, \mathrm{d}$ ). The dissipation phenomenon occurs at high wavenumbers where the smallest vortices dissipate turbulent energy due to viscosity. The dissipation spectra can be estimated as:

$$
D(k)=2 v k^{2} E(k)
$$

where $E(k)$ is Eulerian power spectral densities $\left[\mathrm{m}^{2} \mathrm{c}^{-1}\right]$. While the $k=f \cdot \bar{u}^{-1}$ is spatial wavenumber, where $\bar{u}$ is the average flow velocity at the measuring location.

For non-dimensional coordinates, we used normalization in the form $D(k) / \eta \cdot \varepsilon$ and for wave number $k \cdot \eta$. The measuring point $M$ has a classical form of energy dissipation distribution. The obtained curves in the dissipation interval have a slope $f=1 / 3$. As in the energy spectrum, depending on the angle of attack, they have similar distribution groups. Moreover, the maximum value of dissipation is observed at $\alpha \geq 30^{\circ}$. This pattern clearly correlates with the obtained distributions of the flow velocity behind the airfoil (see Fig. 3 a, d).

\subsection{The structure of the turbulent flow at the point}

To estimate the structure of turbulent flow (stream-wise component) depending on positioning of the measuring cross-section and angle of attack, we chose the point $M$. For this purpose, we used a methodology that is well described in the paper [17]. The authors used correlation time analysis to evaluations the mean temporal and spatial scales of turbulent flows. Where $T_{E}$ and $L_{E}$ characterize the average duration and lifetime of vortices, 
respectively. While the second-order Eulerian longitudinal structure-function was used to estimations the dissipation rate $\epsilon$.

Using the obtained values of $\epsilon$, the dissipation scale $\eta$ and the dissipation time $\tau_{\eta}$ are estimated from classical relations:

$$
\begin{gathered}
\eta=\left(v^{3} / \epsilon\right)^{1 / 4} \\
\tau_{\eta}=(v / \epsilon)^{1 / 2}
\end{gathered}
$$

Also we found the Reynolds number based on the Taylor micro-scale:

$$
R_{\lambda}=\left(\sigma_{u} \lambda\right) / v
$$

where $\lambda=\sigma_{u} \sqrt{15 v / \epsilon}$ is the Taylor micro-scale of the flow. While $\sigma_{u}=\sqrt{N^{-1} \sum_{i=0}^{N-1}\left(u_{i}-\bar{u}\right)^{2}}$ is standard deviation of the streamwise velocity. Accordingly $N$ and $u_{i}$ is the total number of points in the data set and instantaneous velocity at the measuring location, respectively.

The calculated parameters of the turbulent flow at the point M depending on the angle $\alpha$ shown in Fig. 5.
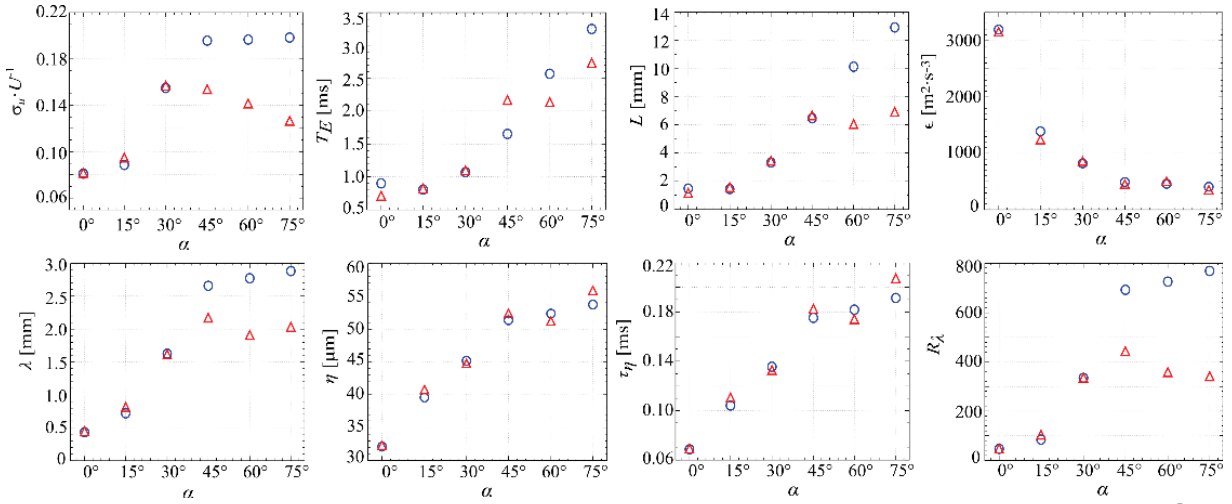

Fig. 5. Characteristics of turbulent flow in point $M$ depending on the angle of attack at $\operatorname{Re} \approx 1.3 \cdot 10^{5}$. The red triangle and the blue circle indicate the measurements at the constant cross-section and at the constant distance, respectively.

The obtained distributions of $T_{E}, \epsilon, \eta$, and $\tau_{\eta}$, regardless of the measuring case, have similar dynamics. Accordingly, their maximum and minimum value at $\alpha=75^{\circ}$ placed around $\epsilon \approx 450$ $\mathrm{m}^{2} \cdot \mathrm{s}^{-3}, \eta \approx 55 \mu \mathrm{m}$ and $\tau_{\eta} \approx 0.2 \mathrm{~ms}$. Whereas at $\alpha \geq 45^{\circ}$, we can see some differences in the distributions of $\sigma_{u} \cdot U^{-1}, L_{E}, \lambda$, and $R_{\lambda}$. The obtained values at the constant distance, in contrast to the constant cross-section, are reduced. For example, in the first and second measuring cases, the value of Taylor microscale Reynolds number at $\alpha=75^{\circ}$ is $R_{\lambda} \approx 800$ and $R_{\lambda} \approx 320$, respectively. A similar trend is also observed for $\sigma_{u} \cdot \mathrm{U}^{-1}$.

It should be noted that the maximum value of the lift coefficient at low Reynolds number for NACA 0012 is observed at $\alpha \approx 45^{\circ}$ [18]. Thus, it is possible to make assumptions about some relationship between the structure of the turbulent flow and the lift coefficient. 


\section{Conclusion}

The wake topology and structure of the turbulent flow behind NACA 0012 airfoil was investigated. Accordingly, the 9116 series NetScanner system and hot-wire anemometry with probe 55P15 were used for this purpose. The experimental studies are conducted at low Reynolds numbers $\operatorname{Re} \approx 1.3 \cdot 10^{5}$ and angles of attack up to $75^{\circ}$ with a step of $15^{\circ}$. Two positions of measuring sections were used in the study. In the first case, with a constant crosssection, the position of which does not change at variations of the angle of attack. Whereas in the second, the distance from the trailing edge to the measuring cross-section was constant. Based on the obtained experimental data, several graphical distributions were obtained to the flow velocity and standard deviation. This allowed us to clearly evaluate the wake topology behind airfoil. We found that regardless of the measurement case, the obtained curves are grouped depending on the angle of attack. Thus, we can conclude that the flowing around the airfoil is better up to $\alpha \leq 15^{\circ}$. A similar trend is observed in the case of analysis of power and dissipation spectra.

To assess the structure of the turbulent flow, the measuring point behind the trailing edge was chosen. Depending on the measuring cases the obtained data have some differences in the distribution of integral length scale, Taylor micro-scale and Reynolds number based on the Taylor micro-scale. The obtained values at $\alpha \geq 45^{\circ}$ and the constant distance, in contrast to the constant cross-section, are reduced.

In general, the analysis showed a certain relationship between the parameters of turbulent flow and the aerodynamic characteristics of the airfoil. The obtained data clearly indicate the position of the optimal angle of attack $\alpha \approx 15^{\circ}$ for the NACA 0012 airfoil.

This work was supported by the project of Technology Agency of the Czech Republic TACR No. TH02020057 "Program Epsilon" and the Internal Doctoral Educational Grant programme (IDEG).

\section{References}

1. Y. Li, J. Wang and P. Zhang, Effects of Gurney Flaps on a NACA0012, Airfoil. Flow, Turbulence and Combustion 68, 27-39 (2002).

2. A. Masayuki, T. Nonomura, H. Aono, A. Oyama, K. Fujii, H. Nagai and K. Asai, Computational and experimental analysis of a high-performance airfoil under lowReynolds-number flow condition, Journal of Aircraft 51, 1864-1872 (2014).

3. F. S. Hover, Ø. Haugsdal, M. S and M. Triantafyllou, Effect of angle of attack profiles in flapping foil propulsion, Journal of Fluids and Structures 19: 1, 37-47 (2004).

4. M. Banafi and M. Banafi, An Experimental research on wake behind airfoil NACA 0012 under various angles, International Journal of Theoretical and Applied Mechanics 2, 164-170 (2017).

5. Ph. Devinant, T. Laverne, J. Hureau, Experimental study of wind-turbine airfoil aerodynamics in high turbulence, Journal of Wind Engineering and Industrial Aerodynamics 90, 689-707 (2000).

6. J. Nedić and J. C. Vassilicos, Vortex Shedding and Aerodynamic Performance of Airfoil with Multiscale Trailing-Edge Modifications, AIAA Journal 53:11, 3240-3250 (2015).

7. B. Zajamšek, C. Doolan and Z. Prime, "Experimental measurements of rotating and stationary wind turbine rotor blade noiseh", 19th Australasian Fluid Mechanics Conference, AFMC (2014). 
8. E. Douvi and D. Margaris, "Aerodynamic characteristics of s809 vs. NACA 0012 airfoil for wind turbine applications", $5^{\text {th }}$ International Conference from Scientific Computing to Computational Engineering, (2012)

9. I. Rodríguez, O. Lehmkuhl, R. Borrell, A. Oliva, Direct numerical simulation of a NACA 0012 in full stall, International Journal of Heat and Fluid Flow 43, 194-203 (2013).

10. V. Yanovych, D. Duda, V. Horáček and V. Uruba, "Research of a wind tunnel parameters by means of cross-section analysis of air flow profiles", AIP Conference Proceedings 2189, (2019).

11. V. Yanovych, D. Duda, V. Horáček and V. Uruba, "Creation of recombination corrective algorithm for research of a wind tunnel parameters", AIP Conference Proceedings 2118, (2019).

12. M. Phillip and T. Kunihiko. "Separation control on NACA 0012 airfoil using momentum and wall-normal vorticity injection", $32^{\text {nd }}$ AIAA Applied Aerodynamics Conference AIAA 2014-2685, (2014).

13. Md. S. Islam, Sh. M. Hakim,M. Alil, and Md. Q. Islam, "Numerical investigation on boundary layer control through moving surface in NACA 0012 airfoil", AIP Conference Proceedings 1851, 020111 (2017).

14. K. Yousefi, R. Saleh, Three-dimensional suction flow control and suction jet length optimization of NACA 0012 wing, Meccanica 50, 1481-1494 (2015).

15. A.N. Kolmogorov, V. Levin, J.C.R. Hunt, O.M. Phillips, D. Williams, Dissipation of energy in the locally isotropic turbulence. Proceedings of the Royal Society of London. Series A: Mathematical and Physical Sciences 434, 15-17 (1991).

16. J. L. Lumley, G. Newman, The return to isotropy of homogeneous turbulence. J. Fluid Meach. 82, 161-178 (1977).

17. V. Yanovych, D. Duda, V. Uruba, Structure turbulent flow behind a square cylinder with an angle of incidence, European Journal of Mechanics, B/Fluids 85, 110-123 (2021).

18. R. E. Sheldahl and P. C. Klimas, "Aerodynamic characteristics of seven symmetrical airfoil sections through 180-degree angle of attack for use in aerodynamic analysis of vertical axis wind turbines", Technical Report, United States, 1981. 